\title{
Broadband Low-Frequency Electroacoustic Absorbers through Hybrid Sensor-/Shunt-Based Impedance Control
}

\author{
Etienne Rivet, Sami Karkar, and Hervé Lissek
}

\begin{abstract}
This paper proposes a hybrid impedance control architecture for an electroacoustic absorber, that combines an improved microphone-based feedforward control with a currentdriven electrodynamic loudspeaker system. Feedforward control architecture enables stable control to be achieved, and current driving method discards the effect of the voice coil inductance. A method is given for designing the transfer function to be implemented in the controller, according to a target specific acoustic impedance and mechanical parameters of the transducer. $\mathrm{Nu}$ merical simulations present the expected acoustic performance, introducing global performance indicators such as the bandwidth of efficient absorption. Experimental assessments in a waveguide confirmed the accuracy of the model and the efficiency of the hybrid control technique for achieving broadband, stable lowfrequency electroacoustic absorbers. An application to damping of resonances in a duct is also presented, and the application to the modal equalization in actual listening rooms is finally discussed.
\end{abstract}

Index Terms-Active sound absorption, electrodynamic loudspeaker, modal equalization, pressure control, resonances damping.

\section{INTRODUCTION}

$\mathbf{I}$ N CRITICAL listening spaces, such as recording studios or home theaters, most problematic room resonances occur within the lowest audible frequency decade [20 - $200 \mathrm{~Hz}$, impairing the quality of sound diffusion in the room within a broad frequency range. Broadband low-frequency sound absorbers are then required to efficiently damp such modes, in order to equalize the room response. However, sound absorption is hardly achievable in the low-frequency range with conventional passive porous materials. Absorbing wedges, which are employed in anechoic chambers, are designed according to the quarter-wavelength rule, leading to a minimal thickness of almost 3 meters at $30 \mathrm{~Hz}$ [1]. Even though the bulkiness can be reduced by a factor 2 by stacking different layers of materials with different flow resistivities [2], the overall thickness of wall-mounted porous absorbers in the low-frequency regime cannot be reduced to just a few meters, which makes them

This work was supported by the Swiss Commission for Technology Innovation (CTI), under grant agreement n14220.1 PFNM-NM.

E. Rivet and H. Lissek are with the Signal Processing Laboratory LTS2, École Polytechnique Fédérale de Lausanne, CH-1015 Lausanne, Switzerland (e-mail: etienne.rivet@epfl.ch; herve.lissek@epfl.ch).

S. Karkar was with the Signal Processing Laboratory LTS2, École Polytechnique Fédérale de Lausanne, CH-1015 Lausanne, Switzerland. He is now with the Tribology and System Dynamics Laboratory (LTDS), École Centrale de Lyon, 69134 Ecully, France (e-mail: sami.karkar@ec-lyon.fr). impractical as furnishing elements for listening rooms. To overcome this size limitation, passive Helmholtz resonators and bass-traps (membrane absorbers) can be used. However, limited by their narrow frequency bandwidth, these passive absorbers are unable to cover the full frequency decade of interest [3].

Active sound absorbers may make it possible to extend the frequency bandwidth over which membrane absorbers present a significant sound absorption capability. The first step towards active sound absorbers consisted in substituting a loudspeaker diaphragm for the passive membrane resonator, and connecting a given electrical impedance or control system to the loudspeaker terminals [4], [5]. This technique for designing electroacoustic absorbers improves both the sound absorption and the bandwidth of the resonator. The shunt impedance was even synthesized to further extend the frequency bandwidth of absorption, whereas achieving perfect sound absorption with a sensorless control loop [6], [7]. The synthesis of an electrical admittance was preferred to that of an impedance since the transfer function of the latter was not proper and could not be implemented on digital platforms. A voltage controlled current source was added to realize the desired electrical impedance at the loudspeaker terminals. Through the synthesized load impedance, the sensorless control required to neutralize the blocked electrical impedance of the voice coil for good sound absorption performance. Several improved models taking into account the semi-inductive behaviour were proposed, but cannot be represented by an equivalent electrical circuit or digitally implemented [8], [9]. Using a simplified model of the blocked electrical impedance of the voice coil in the synthesized impedance deteriorates the sound absorption performance, especially above the loudspeaker resonance frequency.

Alternatively, sensor-based control techniques either require two external sensors [10], [11], but are difficult to use for room applications, or one sensor but requires the design of a constant velocity sound source [12] or the use a velocity estimator [13]. These techniques usually employ a voltage amplifier to drive the loudspeaker, involving the voice-coil inductance in the control, which as a consequence limits the sound absorption performance at higher frequencies. Compensating filters were added in the control loop to counteract the roll-off (due to the voice coil inductance) of the efficient acoustic impedance, improving slightly the robustness and stability of the control 
[14].

Substituting an external sensor for the voltage at the loudspeaker terminals in the shunt impedance appears to be an interesting direction to improve the control stability. Sensorbased control techniques enables sound absorption over a broader bandwidth to be achieved, and the current driving method has the advantage of minimizing the voice coil inductance effect. The motivation of this paper is the development of a hybrid control concept that merges sensor- and shunt-based acoustic impedance control, leading to efficient, broadband low-frequency absorbers.

The paper is organized as follows. In Section II, a model of the electrodynamic loudspeaker is introduced before presenting the hybrid sensor-/shunt-based control, together with a method for identifying control parameters according to a prescribed target specific acoustic impedance. In Section III, numerical simulations are provided, highlighting the wide bandwidth of absorption. Section IV provides an experimental validation of the concept, a discussion on the application to modal damping in a 1-D configuration, and an argumentation on the application of such a concept in reducing the acoustic degradations due to lower order modes in actual listening rooms.

\section{DESIGN OF THE LOW-FREQUENCY ELECTROACOUSTIC} ABSORBER

\section{A. Model of the electrodynamic loudspeaker}

An electrodynamic loudspeaker can be considered as a single-degree-of-freedom oscillator that is mechanically driven by a voice coil within a permanent and almost constant magnetic field. It is assumed that all forces acting on the transducer, especially those resulting from the total sound pressure $p_{t}$ at the diaphragm surface, a combination of the incident and reflected waves, are small enough so that the governing equations remain linear. The mechanical part is assimilated to a simple mass - spring - damper system (i.e., mass $M_{m s}$, mechanical compliance $C_{m s}$ accounting for the surround suspension and the spider, and mechanical resistance $R_{m s}$, respectively) in the low-frequency range.

If we denote the effective piston area by $S_{d}$ and the force factor of the moving-coil transducer by $B l$, the equation of motion of the closed-box loudspeaker diaphragm is derived from Newton's second law, which can be written as

$$
\begin{aligned}
M_{m s} \frac{d v(t)}{d t}= & S_{d} p_{t}(t)-B l i(t)-R_{m s} v(t) \\
& -\left(\frac{1}{C_{m s}}+\frac{\rho c^{2} S_{d}^{2}}{V_{b}}\right) \int v(t) d t
\end{aligned}
$$

where $v$ is the incoming diaphragm velocity and $i$ is the electrical current flowing through the voice coil. The loudspeaker is loaded by a rear cabinet of volume $V_{b}$, the reaction of the fluid acting on the rear face is usually modelled as a mechanical compliance $C_{m b}=V_{b} /\left(\rho c^{2} S_{d}^{2}\right)$, where $\rho$ is the density of the medium and $c$ is the speed of sound in air. The last term of (1) can then be represented by the total mechanical compliance $C_{m c}=\left(C_{m s} C_{m b}\right) /\left(C_{m s}+C_{m b}\right)$ in the following.

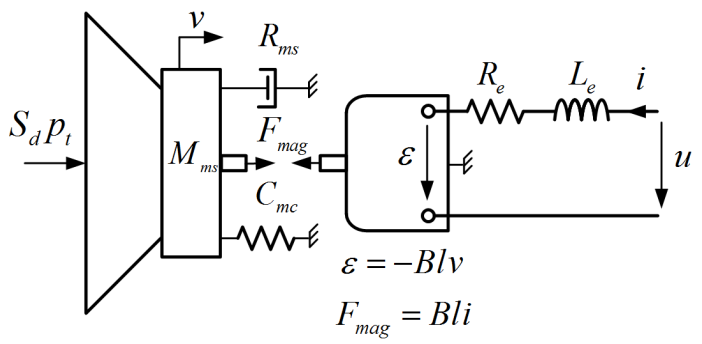

Fig. 1. Schematic of the closed-box electrodynamic loudspeaker.

A simplified governing equation of the electrical dynamics can be written as

$$
u(t)=\left(L_{e} \frac{d}{d t}+R_{e}\right) i(t)+\varepsilon(t)
$$

where $u$ is the input voltage between the electrical terminals, $R_{e}$ is the DC resistance, $L_{e}$ is the self-inductance of the voice coil, and $\varepsilon(t)=-B l v(t)$ is the electromotive force due to the movement of the voice coil within the permanent magnetic field. Fig. 1 shows the schematic of the closed-box electrodynamic loudspeaker.

Considering the Laplace variable $s=j 2 \pi f, f$ being the frequency, the Laplace transform of (1) and (2) reads

$$
\left\{\begin{aligned}
S_{d} P_{t}(s) & =Z_{m}(s) V(s)+B l I(s) \\
U(s) & =Z_{e}(s) I(s)-B l V(s)
\end{aligned}\right.
$$

where $Z_{m}(s)=s M_{m s}+R_{m s}+1 /\left(s C_{m c}\right)$ is the mechanical impedance of the closed-box loudspeaker and $Z_{e}(s)=s L_{e}+$ $R_{e}$ is the blocked electrical impedance of the voice coil.

\section{B. Sound absorption capability}

The dynamic response of the diaphragm to an external acoustic disturbance, characterizing the acoustic properties of the surface, can be described from the specific acoustic impedance, which is defined as the complex ratio of the total sound pressure $P_{t}(s)$ at the diaphragm to the diaphragm velocity $V(s)$. In the case of the open circuit loudspeaker, namely the case where no electrical current $i$ circulates through the coil, this quantity can be directly derived from (3a) as

$$
Z_{s}(s)=\frac{P_{t}(s)}{V(s)}=\frac{Z_{m}(s)}{S_{d}}
$$

For 1-D configurations, the sound absorption coefficient, which defines the ratio of the acoustic energy absorbed by the loudspeaker over the incident energy (under normal incidence), is expressed as

$$
\alpha(f)=1-\left|\frac{Z_{s}(f)-\rho c}{Z_{s}(f)+\rho c}\right|^{2}
$$

The bandwidth of efficient sound absorption is defined as the frequency range over which the total sound energy in front of the diaphragm is less than twice the total sound energy in the ideal case $\alpha=1$, that is to say $p_{t} \leq \sqrt{2} p_{i}$. This criterion 




(a)

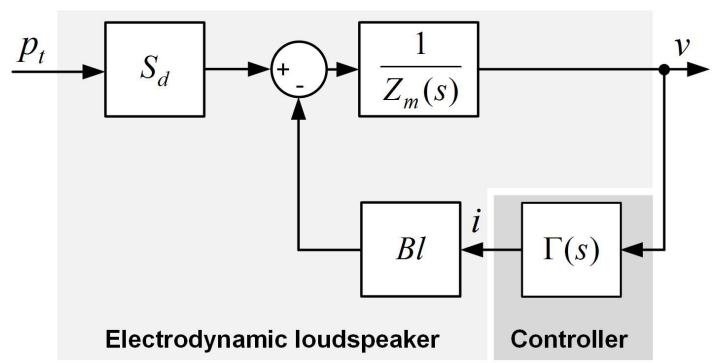

(b)

Fig. 2. Block diagrams of the electrodynamic loudspeaker system under control by sensing (a) the total sound pressure at the diaphragm or (b) diaphragm velocity. The light grey and dark grey areas highlight the plant and controller respectively.

corresponds to a sound absorption coefficient $\alpha \geq 0.83^{1}$. Note that the sound absorption capability of the loudspeaker diaphragm can be modified through the control of the electrical current circulating through the voice-coil, as can be seen in (3a).

\section{Formulation of the hybrid sensor-/shunt-based control con- cept}

The main motivation of the method presented in this paper is to be able to design stable broadband low-frequency electroacoustic absorbers, with the aim of practical implementation of room modal equalization (with acoustic and/or electroacoustic sources) and noise control in buildings. No prior information about the sound source (signal, location) is known from the system. Simple models of the blocked electrical impedance, as expressed in Eq. (3b), do not take into account the semiinductive behaviour accurately enough. This mismatch causes a limitation for the achieved absorption bandwidth in the synthesized shunt impedance control [7]. Improved models, as those proposed in [8] and [9], cannot be represented by an equivalent electrical circuit or digitally implemented. Moreover, voltage drive sensor-based techniques unavoidably involve the voice-coil inductance in the control, which, as a consequence, also limits the sound absorption performance at higher frequencies. The current driving method makes it possible to bypass (3b), minimizing the voice coil inductance effect. Using only one sensor and taking into account the loudspeaker model involved in (3a), as in the method presented

\footnotetext{
${ }^{1}$ Ideal case: the total sound energy is equal to the incident wave energy. Threshold case: the total sound energy in front of the diaphragm (sound energy of the sum of incident and reflected waves) is equal to twice the sound energy of the incident wave alone.
}

in [7], it is possible to control the dynamic response of the diaphragm of the current-driven loudspeaker. Assuming that a target specific acoustic impedance $Z_{s t}$ is realized at the diaphragm, two approaches are presented in the following, depending on whether a pressure or velocity sensor is used.

1) First approach: from total sound pressure to electrical current: The transfer function $\Theta(s)$ from the total sound pressure $P_{t}(s)$ at the diaphragm to the electrical current $I(s)$ can be derived from (3a) as

$$
\Theta(s)=\frac{I(s)}{P_{t}(s)}=\frac{S_{d} Z_{s t}(s)-Z_{m}(s)}{B l Z_{s t}(s)}
$$

The closed form expression of the specific acoustic impedance then becomes

$$
Z_{s}(s)=\frac{Z_{m}(s)}{S_{d}-B l \Theta(s)}
$$

Figure 2a shows the block diagram of the controlled loudspeaker by sensing the total sound pressure at the diaphragm. In this scheme, it can be clearly seen that the blocked electrical impedance $Z_{e}$ of the loudspeaker is absent from the control. Current drive presents the advantage of discarding Kirchhoff's law (2), thus eliminating the blocked electrical impedance $Z_{e}$ in the equation of the loudspeaker, whereas this quantity can potentially be a source of instability in a voltage drive control configuration [10], [14]. Nevertheless, this strategy requires an accurate evaluation of the loudspeaker mechanical parameters, such as the effective piston area $S_{d}$, the force factor $B l$, and the mechanical impedance (mass $M_{m s}$, resistance $R_{m s}$, and compliance $C_{m c}$ ).

2) Second approach: from diaphragm velocity to electrical current: A similar approach can be followed by sensing the diaphragm velocity instead of the total sound pressure. The transfer function $\Gamma(s)$ from the diaphragm velocity $V(s)$ to the electrical current $I(s)$ yields

$$
\Gamma(s)=\frac{I(s)}{V(s)}=\frac{S_{d} Z_{s t}(s)-Z_{m}(s)}{B l}
$$

The specific acoustic impedance thus takes the form

$$
Z_{s}(s)=\frac{Z_{m}(s)+B l \Gamma(s)}{S_{d}}
$$

Fig. 2b shows the block diagram of the controlled loudspeaker by sensing the diaphragm velocity, thus taking the form of a feedback control architecture.

3) Control strategy: Depending on the expression of the target specific acoustic impedance $Z_{s t}(s)$ and due to real-time constraints at low frequencies, one approach may be more appropriate than the other. As the use of a pressure sensor is less expensive and easier to implement than a velocity sensor, the implementation of the transfer function $\Theta(s)$ has been preferred to that of $\Gamma(s)$ in the following.

Discarding the electrical part of the loudspeaker and using the signal received from a sensor thus allows for a simple way to control the dynamic response of the diaphragm to an external sound pressure, by modifying its mechanical resistance and reactance simultaneously. 


\section{Target specific acoustic impedance for the diaphragm}

To achieve maximal sound absorption under normal incidence (i.e. $\alpha=1$ ), the specific acoustic impedance $Z_{s}(s)$ may ideally be set to a target specific acoustic resistance $R_{s t}$ equal to the characteristic specific acoustic impedance of the medium $Z_{c}=\rho c$ (corresponding to the impedance matching case). This implies that the phase between the total sound pressure $P_{t}(s)$ at the diaphragm and diaphragm velocity $V(s)$ should be zero over the whole frequency range. However, the specific acoustic impedance $Z_{s}(s)$ in (4) includes reactive terms due to the compliance $C_{m c}$ and mass $M_{m s}$ of the diaphragm, which invariably induces a mismatch with the desired specific acoustic resistance $R_{s t}$ away from the resonance frequency. As neither the mass nor the compliance can be completely cancelled, a practical solution is to define a complex, frequency-dependent target specific acoustic impedance by the following parametric model:

$$
Z_{s t}(s)=s \frac{\mu M_{m s}}{S_{d}}+R_{s t}+\frac{\mu}{s S_{d} C_{m c}}
$$

where $0<\mu<1$ is a factor that decreases simultaneously the effective mass $\mu M_{m s}$ and stiffness $\mu / C_{m c}$, in order to extend the bandwidth of maximal sound absorption. The corresponding resonance frequency is equal to

$$
f_{0}=\frac{1}{2 \pi \sqrt{M_{m s} C_{m c}}}
$$

In this configuration, the bandwidth of efficient sound absorption is found to be

$$
B W=\frac{S_{d}}{2 \pi \mu M_{m s}} \sqrt{\frac{(\sqrt{2}-1)^{2}\left(R_{s t}+Z_{c}\right)^{2}-\left(R_{s t}-Z_{c}\right)^{2}}{1-(\sqrt{2}-1)^{2}}}
$$

and is only valid for

$$
\left|R_{s t}-\sqrt{2} Z_{c}\right| \leq Z_{c}
$$

Note that the higher the term $S_{d} / M_{m s}$, the wider the bandwidth $B W$. Moreover, the compliance $C_{m c}$ is not involved in (12), but makes it possible to adjust passively the desired resonance frequency $f_{0}$ of the low-frequency electroacoustic absorber, through the cabinet volume $V_{b}$ as can be seen in (2) and (11). The control of parameter $\mu$ then extends the bandwidth of efficient sound absorption around this central frequency. It is also conceivable to apply two different reduction factors $\mu_{1}$ for the mass and $\mu_{2}$ for the stiffness in (10), to shift the resonance frequency $f_{0}$ without modifying the cabinet volume $V_{b}{ }^{2}$.

\section{NUMERICAL ANALYSIS}

\section{A. Sound absorption performance}

The overall performance of the electroacoustic absorber was first evaluated by computing the equations presented in Section II, considering a Peerless SDS-P830657 loudspeaker mounted in a closed-box of volume $V_{b}=10 \mathrm{dm}^{3}$. The mechanical parameters of the loudspeaker were estimated from the measurement of the mechanical impedance of the loudspeaker diaphragm, mounted at the termination of a standing-wave duct,

\footnotetext{
${ }^{2}$ This case will not be further investigated here.
}

TABLE I

Small Signal Parameters of the Peerless SDS-P830657 LOUdSPEAKER IN A CLOSED-BoX OF VOLUME $V_{b}=10 \mathrm{DM}^{3}$

\begin{tabular}{lccc}
\hline \hline Parameter & Notation & Value & Unit \\
\hline Effective piston area & $S_{d}$ & 151 & $\mathrm{~cm}^{2}$ \\
Moving mass & $M_{m s}$ & 14.67 & $\mathrm{~g}$ \\
Mechanical resistance & $R_{m s}$ & 1.31 & $\mathrm{~N} \cdot \mathrm{s} \cdot \mathrm{m}^{-1}$ \\
Mechanical compliance & $C_{m c}$ & 242.35 & $\mu \mathrm{m} \cdot \mathrm{N}^{-1}$ \\
Force factor & $B l$ & 5.98 & $\mathrm{~N} \cdot \mathrm{A}^{-1}$ \\
Density of the air at $294 \mathrm{~K}$ & $\rho$ & 1.2 & $\mathrm{~kg} \cdot \mathrm{m}^{-3}$ \\
Sound speed in the air at $294 \mathrm{~K}$ & $c$ & 344 & $\mathrm{~m} \cdot \mathrm{s}^{-1}$ \\
\hline \hline
\end{tabular}

TABLE II

Setting CASEs And CorResponding Computed Control Results

\begin{tabular}{cccccc}
\hline \hline & \multicolumn{2}{c}{ Setting cases } & & \multicolumn{2}{c}{ Control results } \\
\cline { 2 - 3 } \cline { 5 - 6 } & Reduction factor & Target resistance & & Resonance frequency & Bandwidth \\
& $\mu$ & $R_{s t}\left(\mathrm{~Pa} \cdot \mathrm{s} \cdot \mathrm{m}^{-1}\right)$ & & $f_{0}(\mathrm{~Hz})$ & $B W(\mathrm{~Hz})$ \\
\hline $\mathrm{A}$ & 1 & $R_{m s} / S_{d}$ & & 84 & - \\
$\mathrm{B}$ & 0.15 & $\rho c / 8$ & & 84 & - \\
$\mathrm{C}$ & 0.15 & $\rho c$ & & 84 & 410 \\
\hline \hline
\end{tabular}

excited by an external sound source with broadband noise. The experimental setup is further detailed in Section IV. This way, the acoustic radiation impedance which depends on the environment in which the loudspeaker is located, was already taken into account in the mechanical impedance $Z_{m}$. To avoid numerous annotations, the mechanical impedance components (i.e. $M_{m s}, R_{m s}$, and $C_{m c}$ ) account for the radiation impedance in the following. The physical parameters are reported in Table I.

Table II presents three simulation cases (labelled A-C) corresponding to three sets of values for the target specific acoustic impedance parameters $\left(\mu\right.$ and $R_{s t}$ ). Although the proposed control strategy makes it possible to change the resonance frequency of the electroacoustic absorber, all simulated cases have the same resonance frequency. The last two columns of Table II present the achieved control performance in terms of resonance frequency $f_{0}$ and bandwidth $B W$ of efficient absorption. The baseline configuration A corresponds to the passive mechanical resonator situation, where the loudspeaker was in open circuit (no electrical current flowing through the voice coil). Configurations $\mathrm{B}$ and $\mathrm{C}$ correspond to control settings achieving the same diminution of $85 \%$ of the loudspeaker effective mass and stiffness, and assigning the target acoustic resistances $\rho c / 8$ and $\rho c$ respectively.

Fig. 3a illustrates the frequency responses of the specific acoustic impedances computed from the control setting values listed in Table II. The expected performance in terms of sound absorption coefficient is given in Fig. $3 \mathrm{~b}$. When the loudspeaker is in open circuit (case A), the electroacoustic absorber behaves as a passive second-order bandpass system. At low frequencies the specific acoustic impedance is controlled by stiffness, the magnitude decreasing as $1 /\left(S_{d} C_{m c} f\right)$. Then it is controlled by resistance $R_{m s} / S_{d}$, with a minimum 

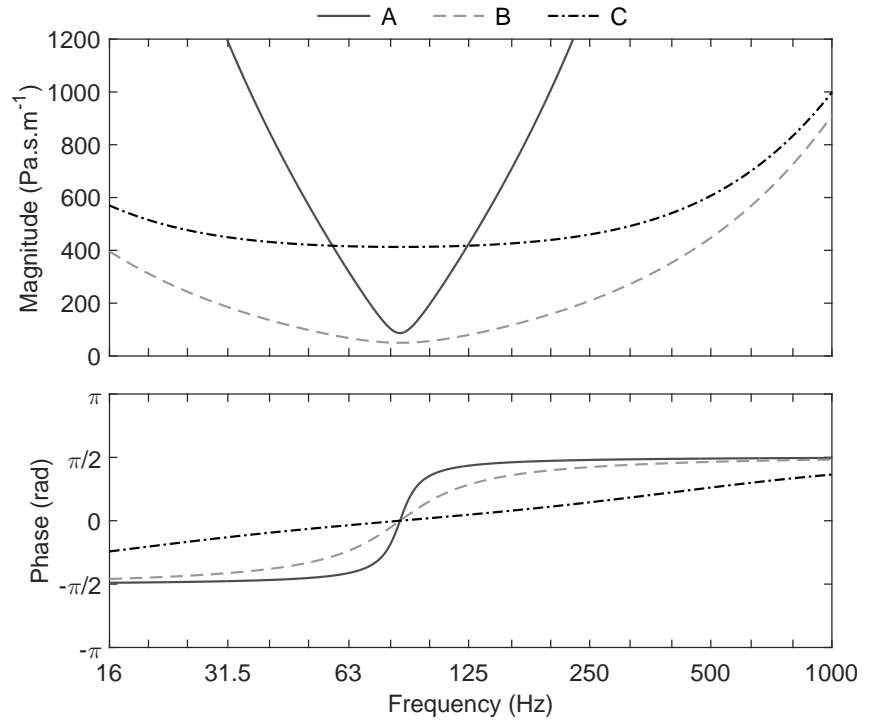

(a)

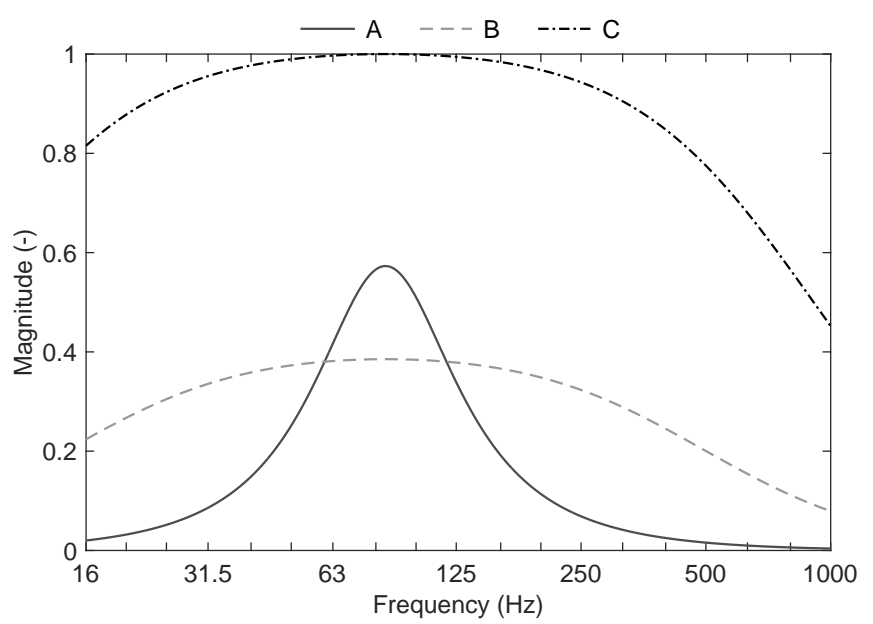

(b)

Fig. 3. (a) Bode plots of the specific acoustic impedances and the corresponding sound absorption coefficients (b) of the electroacoustic absorber computed in open circuit (case A) and under control (cases B and C).

amplitude at resonance, and above resonance it is controlled by mass, the magnitude increasing as $f M_{m s} / S_{d}$. The phase shift between the total sound pressure at the diaphragm and diaphragm velocity tends to $-\pi / 2$ at low frequencies, is equal to zero at resonance, and tends to $\pi / 2$ at high frequencies. Case $\mathrm{C}$ in Fig. 3a shows that the specific acoustic impedance of the diaphragm could be matched to the characteristic impedance of the medium $\rho c$ over a large frequency range, whereas decreasing the mass and stiffness by $85 \%$ relative to those presented by the passive loudspeaker diaphragm, thus extending the control bandwidth. In case B, the target acoustic resistance value was chosen so as to be smaller than the one obtained with the passive loudspeaker diaphragm, to illustrate the possibility to assign small values of acoustic resistance with this control strategy. As shown in Fig. 4, the bode plots of the transfer functions $\Theta(s)$ computed for cases B and C have different quality factors, according to the desired specific acoustic resistance $R_{s t}$. The phase of the transfer function also
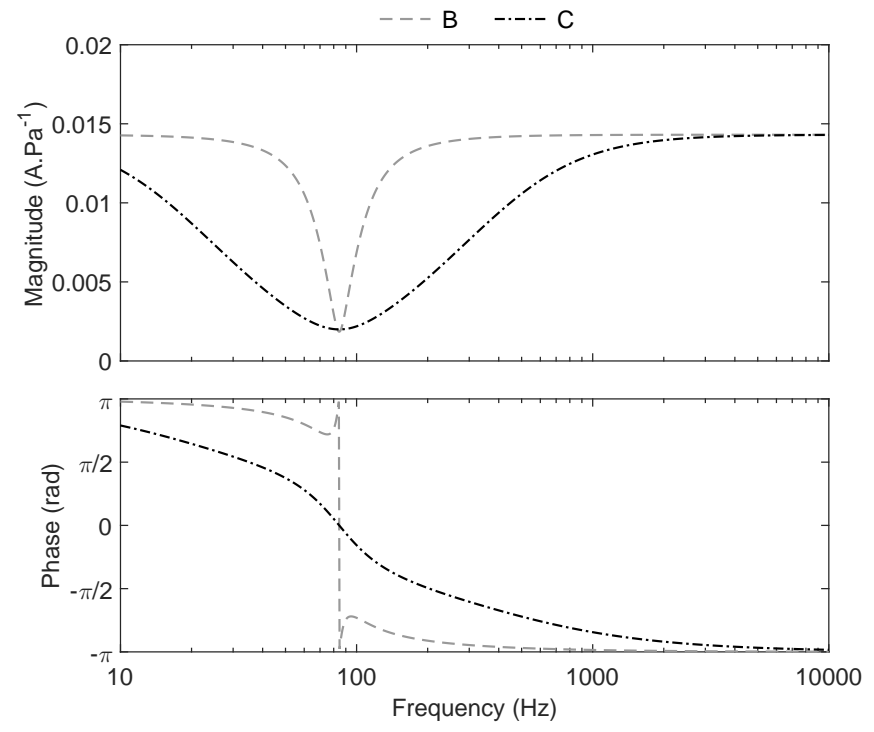

Fig. 4. Bode plots of the transfer function $\Theta(s)$ from the total sound pressure at the diaphragm to the current flowing through the voice coil computed for the setting cases $\mathrm{B}$ and $\mathrm{C}$.

changes around the resonance frequency depending on the sign of $S_{d} R_{s t}-R_{m s}$.

\section{B. Damping of low-frequency resonances}

To illustrate the capability of the broadband electroacoustic absorber to damp low-frequency resonances in a waveguide, transfer functions from the driving voltage of the sound source to the sound pressure level were processed along the duct, for different terminations of the duct. The sound pressure levels with the hard-wall condition were compared to the cases where an electroacoustic absorber terminated the duct, either in open-circuit (case A) or with the control (case C). Through the simulation, it is intended to show the effect of the active impedance control, both in terms of sound absorption performance and attenuation of duct modes. A sound source is located at the left end of the duct of length $L=1.97 \mathrm{~m}$ ${ }^{3}$. It consists of a voltage-driven loudspeaker in a closed-box of volume $V_{b}=10 \mathrm{dm}^{3}$ with physical parameters summarized in Table III (see Appendix A). The duct section $S$ is equal to the effective piston area $S_{d}$ of the electroacoustic absorber and to that of the sound source loudspeaker, to simplify the analytical study. If the absorber area was substantially smaller than the cross-section of the waveguide, the hypothesis of a uniform pressure at the boundary would not be valid anymore. The analytical approach would require the total decomposed field on transverse modes [15], whereas a practical approach could be done with the help of a Finite Element Method software [16]. A surface impedance condition is imposed at the right end of the waveguide. In one case, to simulate the rigid termination, the impedance is only resistive on the whole frequency range and is equal to $16 \mathrm{kPa} \cdot \mathrm{s} \cdot \mathrm{m}^{-1}$, which amounts to a sound absorption coefficient $\alpha \simeq 0.1$. In the two other configurations, the specific acoustic impedance corresponds to

\footnotetext{
${ }^{3}$ The parameter was adjusted to be in agreement with the experimental setup presented in Section IV.
} 


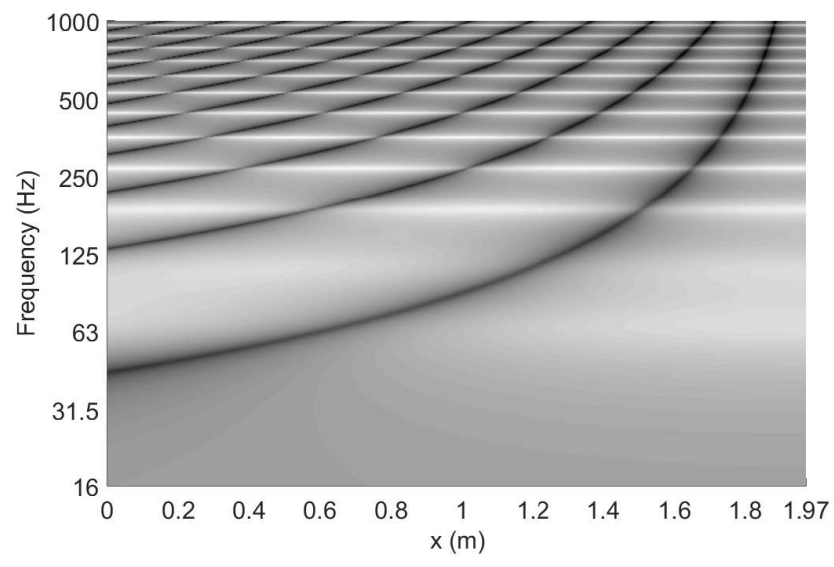

(a)

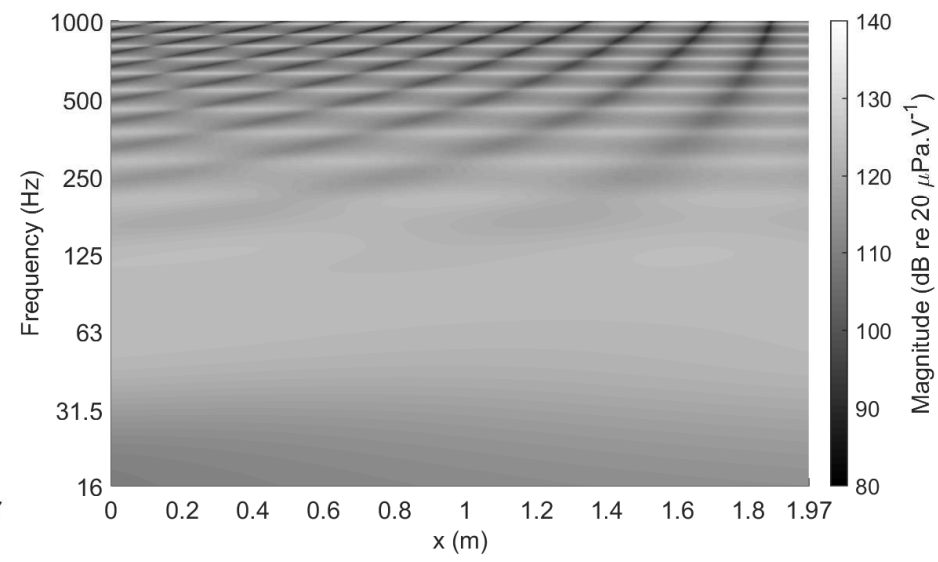

(b)

Fig. 5. Space-frequency maps of transfer functions from the driving voltage of the sound source located at the left end of the waveguide to the sound pressure level. At the right end is imposed (a) hard-wall termination or (b) electroacoustic absorber with the control settings of case C.

(10) with setting cases A (open circuit) and C (with control) listed in Table II.

Given a surface impedance $Z_{s_{L}}$ at location $x=L$, the sound pressure satisfying the Helmholtz equation can be expressed as

$$
p(x)=a\left(e^{-j k x}+r_{L} e^{j k(x-2 L)}\right)
$$

where $j^{2}=-1, k=2 \pi f / c$ is the wavenumber, $r_{L}=\left(Z_{s_{L}}-\right.$ $\rho c) /\left(Z_{s_{L}}+\rho c\right)$ is the reflection coefficient at location $x=L$, and $a$ is a coefficient depending on the surface impedance $Z_{s_{L}}$, physical parameters and driving voltage of the sound source (see Appendix A for more details). As shown in [16], in the case of a hard-wall termination, the resonance frequencies are equal to

$$
f_{r_{n}}=n \frac{c}{2 L}
$$

where $n \in \mathbb{N}$. The anti-resonance frequencies $f_{a r_{n}}$ can be computed from (14) when $p(0)=0$. Simplifying $\left(Z_{s_{L}}-\right.$ $\rho c) /\left(Z_{s_{L}}+\rho c\right)=1$ at any frequency $f$, nulls of pressure occur at locations

$$
x_{a r_{n}}(f)=L-(2 n+1) \frac{c}{4 f}
$$

Anti-resonance frequencies are thus found inverting (16) for $x_{a r_{n}}=0$ :

$$
f_{a r_{n}}=(2 n+1) \frac{c}{4 L}
$$

Fig. 5 shows space-frequency maps of transfer functions from the driving voltage of the sound source located at the left end of the waveguide to the sound pressure level, expressed in $\mathrm{dB}$ re. $20 \mu \mathrm{Pa} \cdot \mathrm{V}^{-1}$, when the right end is closed either by a hard-wall or by the electroacoustic absorber (case $\mathrm{C}$ ). As can be seen in Fig. 5a, the sound field is characterised by an uneven acoustic energy distribution at low frequencies when the waveguide is closed by a hard-wall. The strong resonances (in white) appear at fixed frequencies whatever the location in the waveguide, whereas the nulls (in black) are shifted as the microphone position moves away from the sound source as indicated in (16). When the electroacoustic absorber substitutes for the hard wall (Fig. 5b), both peaks (resonances) and dips (nulls) in frequency spectra are significantly attenuated with the active control settings of case C. Additional figures can be found in Appendix B. This analytical study reveals the performance of the electroacoustic absorber for the modal equalization in a waveguide over a certain frequency bandwidth.

\section{EXPERIMENTAL RESULTS AND DISCUSSION}

\section{A. Experimental setup and control system implementation}

To experimentally validate the preceding results, a waveguide was designed (length $L=1.97 \mathrm{~m}$ and internal diameter $\phi=150 \mathrm{~mm}$ ) as depicted in Fig. 6. Both terminations were closed by electrodynamic loudspeakers in closed boxes of volume $V_{b}=10 \mathrm{dm}^{3}$, as presented in Section III. The sound source at the left termination delivered a broadband pink noise, whose the bandwidth was $[2 \mathrm{~Hz}-20 \mathrm{kHz}]$. The specific acoustic impedance and sound absorption coefficient were assessed according to ISO 10534-2 standard [17]. Three 1/2" microphones (Norsonic Type 1225 cartridges mounted on Norsonic Type 1201 amplifier) were wall-mounted at positions $x_{1}=1.02 \mathrm{~m}$, $x_{2}=1.51 \mathrm{~m}$, and $x_{3}=1.62 \mathrm{~m}$ from the sound source, sensing the sound pressures $p_{1}=p\left(x_{1}, t\right), p_{2}=p\left(x_{2}, t\right)$, and $p_{3}=p\left(x_{3}, t\right)$. The transfer functions $H_{13}=p_{3} / p_{1}$ and $H_{23}=p_{3} / p_{2}$ were processed through a Brüel and Kjær Pulse multichannel analyser. This experimental setup enabled the electroacoustic absorber performance to be assessed under normal incident plane waves, over a frequency range between $44-1340 \mathrm{~Hz}$. The displayed frequency range was reduced to 1 $\mathrm{kHz}$ to focus the analysis on the bandwidth over which the absorption was supposed to be efficient, according to Table II.

The pressure used in the control was sensed with a 130D20 ICP microphone, located at $1 \mathrm{~cm}$ from the electroacoustic absorber diaphragm and close to the lateral duct wall as depicted in Fig. 6. The transfer function $\Theta(s)$ given by (6) was first discretized in a discrete-time recursive filter, and then was implemented onto a real-time National Instruments 


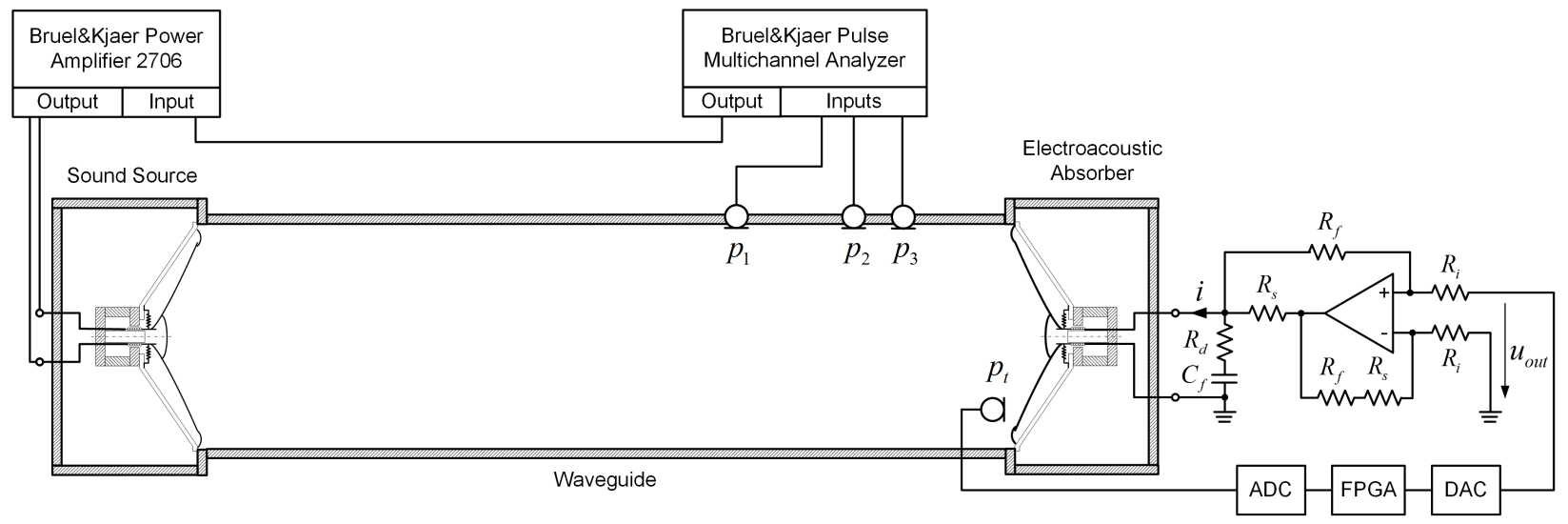

Fig. 6. Scheme of the experimental setup. The control implementation is depicted in the right-hand side including the microphone, the digital controller, and the transconductance amplifier.
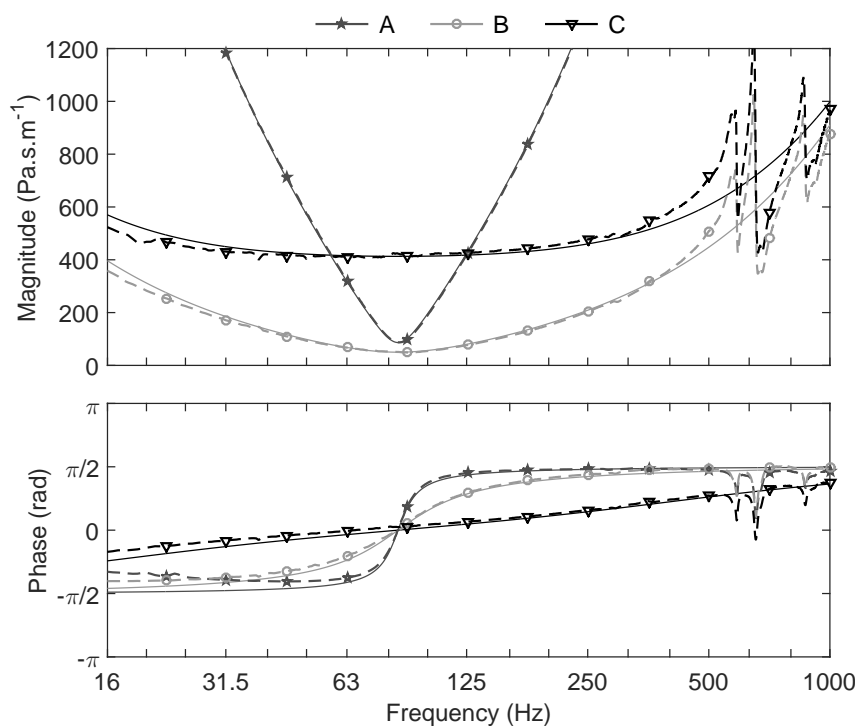

(a)

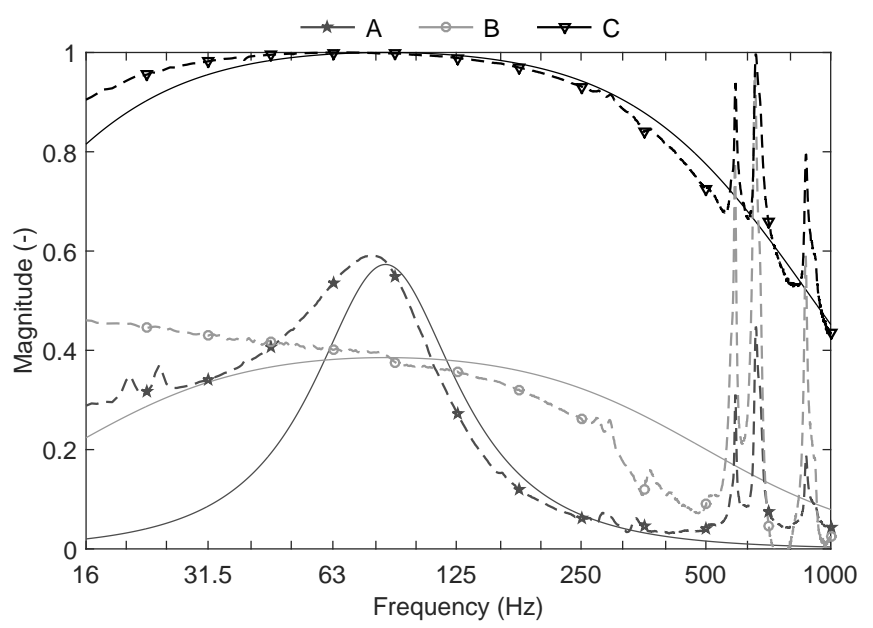

(b)

Fig. 7. (a) Frequency responses of the specific acoustic impedances and (b) corresponding sound absorption coefficients of the electroacoustic absorber measured (dotted lines) and simulated (solid lines) in open circuit (case A) and under control (cases B and C).
CompactRIO platform supporting FPGA technology ${ }^{4}$. The voltage signal from the microphone was digitally converted thanks to an analog module NI9215. The output filtered signal $u_{\text {out }}$ was delivered by an analog module NI 9263 to a voltage controlled current source that drove the voice coil loudspeaker. As illustrated on the right-hand side in Fig. 6, the voltage controlled current source was an op-amp based "improved" Howland current pump circuit [18], including an operational amplifier, two input resistors $R_{i}$, two feedback resistors $R_{f}$, a current sense resistor $R_{s}$. As the load was reactive, a compensation circuit supplied by a resistance $R_{d}$ and capacitance $C_{f}$ was added to ensure stability with the grounded load [19].

\section{B. Sound absorption measurements}

The setting cases used for the measurements were the same as those used for running the simulation in Section III, and are summarized in Table II. Figure 7 illustrates the performance of the electroacoustic absorber in terms of sound absorption, through the measured frequency response of the specific acoustic impedance and the corresponding sound absorption coefficient. These results show that the measurements are satisfactorily consistent with the corresponding simulation (see Fig. 3). As expected, we obtained a perfect acoustic absorption over a broad frequency range around the natural resonance of the loudspeaker. With this control strategy, both the acoustic resistance and reactance of the diaphragm were modified, to reach as close as possible the desired specific acoustic impedance $Z_{s t}$. The slight differences can be attributed to imperfections in the lumped parameter model and to the frequency response function of the microphone used in the control loop, which was not taken into account in the control. The resonances at $587 \mathrm{~Hz}$ and $655 \mathrm{~Hz}$ are due to the first modes of the electroacoustic absorber enclosure. As discussed in Section II, it seems obvious that the target specific acoustic impedance can not be reduced to a constant resistive value, due to the reactive terms of the diaphragm. The value of the reduction factor $\mu$ is limited by the stability of the closed

\footnotetext{
${ }^{4}$ The microphone sensitivity and gain of the voltage controlled current source were included in the transfer function $\Theta(s)$.
} 


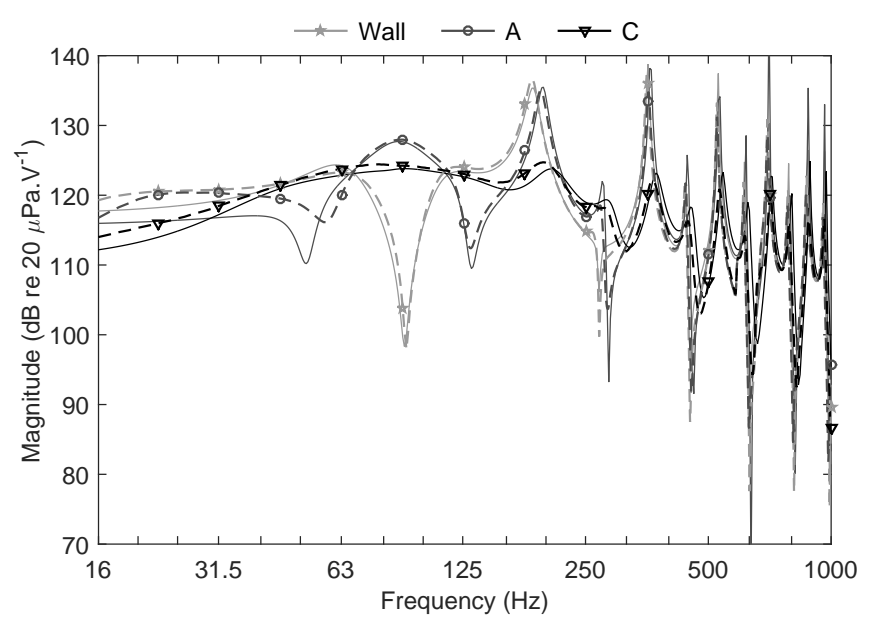

(a)

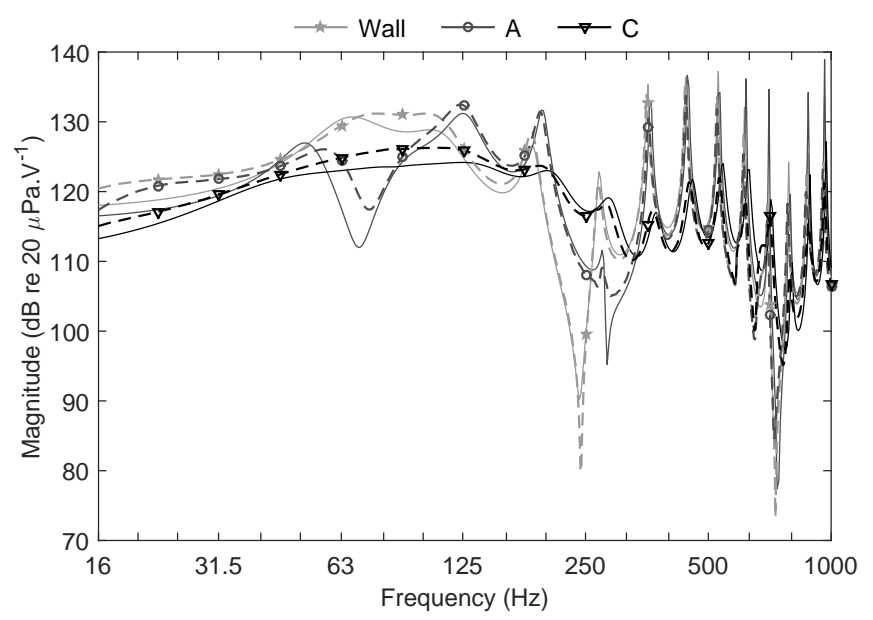

(b)

Fig. 8. Transfer functions from the driving voltage of the sound source located at the left end of the waveguide to the sound pressure level, measured (dotted lines) and simulated (solid lines), at locations (a) $x_{1}=1.02 \mathrm{~m}$ and (b) $x_{3}=$ $1.62 \mathrm{~m}$ from the sound source. At the right end was imposed a hard-wall termination or an electroacoustic absorber in cases $\mathrm{A}$ or $\mathrm{C}$.

loop system, between the electroacoustic absorber and the environment in which it is set up. The stability robustness may also be reduced if some characteristics of the loudspeaker dynamics are not modelled, as the diaphragm modal behaviour for instance.

\section{Modal equalization in a waveguide}

The control performance for damping low-frequency modal resonances were then assessed in the waveguide. Figure 8 shows the measured transfer functions from the driving voltage of the sound source located at the left end of the waveguide to the sound pressure level, expressed in $\mathrm{dB}$ re. $20 \mu \mathrm{Pa} \cdot \mathrm{V}^{-1}$, when the duct was ended by a hard wall and by the electroacoustic absorber in cases $\mathrm{A}$ and $\mathrm{C}$. The measurements are in good agreement with the corresponding simulation. The study focused on the global acoustic benefit at low frequencies. From $44 \mathrm{~Hz}$ up to $300 \mathrm{~Hz}$, the difference of sound pressure level between the peaks and dips was $51.3 \mathrm{~dB}$ for the rigid termination, droped to $37.3 \mathrm{~dB}$ for the open circuit loudspeaker system (case A), and was reduced down to $12.6 \mathrm{~dB}$ for the active electroacoustic absorber (case $\mathrm{C}$ ). Reducing the sound pressure level dynamics by a ratio of 4 to 1 , these results reveal the modal damping efficiency of the electroacoustic absorber over a certain frequency bandwidth. The magnitudes of resonances and anti-resonances were therefore minimized thanks to the active electroacoustic absorber, thereby equalizing the distribution of sound energy both along the waveguide and the frequency axis.

\section{Application to rooms}

In the case of rooms, this effect of modal equalization obtained in the waveguide is particularly desired in listening spaces, such as recording studios or home theaters, to improve the sound quality. Changes must be made for room applications, compared to what was presented in the previous sections. First, analysing the acoustic energy distribution of rooms with specific geometry makes it possible to know where the pressure nodes are, to indicate where to place electroacoustic absorbers for optimal performance (in corners for plane-parallel rooms). Then the optimal target specific acoustic resistance can be different from the characteristic specific acoustic impedance of the air and can vary from one mode to another [16]. As each mode has its own central frequency, an optimal absorber would have a frequency-dependent specific acoustic resistance that matches these different values. Another form of target specific acoustic impedance could then be preferred to that given by (10). Finally for practical reasons, the total absorbing area should be relatively small compared to the total reflecting area of the room. Preliminary studies showed promising results to damp resonances in several rooms using these electroacoustic absorbers.

\section{CONCLUSION}

This paper presented a novel control architecture to achieve stable broadband low-frequency sound absorption. The technique results from a sensor-/shunt-based acoustic impedance control, combined with a current amplifier in a similar fashion as recent sensorless acoustic impedance synthesis control techniques. The main advantage of driving the electroacoustic absorber with a prescribed electrical current is to minimize the negative effects of the voice coil inductance on the control stability above the resonance frequency. Numerical analysis and experimental assessment confirmed the improvement of performance compared to the absorption techniques reported so far, especially in terms of stability and extension of bandwidth. The technique stands out for its low sensitivity to the voice coil inductance, which can limit the performance of conventional electroacoustic absorbers techniques. An application to duct mode damping over a significantly broad frequency range confirmed the efficiency of the electroacoustic absorber to equalize the acoustic response in cavities. Further studies will investigate the actual application of such hybrid sensor/shuntbased electroacoustic absorber to rooms, and should confirm the efficiency of the concept to damp the low-frequency resonances in rooms. 
TABLE III

SMall Signal Parameters of THe PeERLESS SDS-P830657 Loudspeaker of THE SOUND SOURCE IN A Closed-Box of Volume $V_{b}=10 \mathrm{DM}^{3}$

\begin{tabular}{lccc}
\hline \hline Parameter & Notation & Value & Unit \\
\hline Effective piston area & $S_{d}$ & 151 & $\mathrm{~cm}^{2}$ \\
Moving mass & $M_{m s_{s}}$ & 12.9 & $\mathrm{~g}$ \\
Mechanical resistance & $R_{m s_{s}}$ & 1.23 & $\mathrm{~N} \cdot \mathrm{s} \cdot \mathrm{m}^{-1}$ \\
Mechanical compliance & $C_{m c_{s}}$ & 260.79 & $\mu \mathrm{m} \cdot \mathrm{N}^{-1}$ \\
Force factor & $B l_{s}$ & 5.98 & $\mathrm{~N} \cdot \mathrm{A}^{-1}$ \\
Voice coil inductance & $L_{e_{s}}$ & 0.38 & $\mathrm{mH}$ \\
DC resistance & $R_{e_{s}}$ & 6 & $\Omega$ \\
\hline \hline
\end{tabular}

\section{APPENDIX A}

\section{ANALYTICAL MODELING OF THE EXPERIMENTAL SETUP}

The physical parameters of the sound source loudspeaker are summarized in Table III. The mechanical parameters of the loudspeaker were estimated from the measurement of the mechanical impedance, as explained in Section III, and its electrical parameters were simply retrieved from the manufacturer's data.

Denoting the blocked electrical impedance and the mechanical impedance of the sound source by $Z_{e_{s}}$ and $Z_{m_{s}}$, the sound pressure satisfying Helmholtz's equation in the waveguide is given by (14), with

$$
a=\frac{\rho c}{S_{d} \rho c\left(1+r_{L} e^{-2 j k L}\right)+Z_{m_{0}}\left(1-r_{L} e^{-2 j k L}\right)} \frac{B l_{s}}{Z_{e_{s}}} U_{s}
$$

where $U_{s}$ is the driving voltage of the sound source and $Z_{m_{0}}=$ $Z_{m_{s}}+\left(B l_{s}\right)^{2} / Z_{e_{s}}$ is the equivalent mechanical impedance of the sound source at location $x=0$.

\section{APPENDIX B}

\section{ADDITIONAL FIGURES OF THE NUMERICAL ANALYSIS}

Fig. 9 shows a space-frequency map of the transfer function from the driving voltage of the sound source located at the left end of the waveguide to the sound pressure level, expressed in $\mathrm{dB}$ re. $20 \mu \mathrm{Pa} \cdot \mathrm{V}^{-1}$, when the right end is closed by the electroacoustic absorber in open circuit (case A).

Fig. 10 shows the superposition of the transfer functions between the sound source driving voltage at the left end of the waveguide and the sound pressure at all every location along the waveguide, expressed in $\mathrm{dB}$ re. $20 \mu \mathrm{Pa} \cdot \mathrm{V}^{-1}$. These figures were obtained from the normal projection on the frequency and magnitude axes planes, illustrated in Figs. 5 and 9, according to the configuration at the right end of the waveguide: hardwall termination, electroacoustic absorber in open circuit, and under control in case $\mathrm{C}$, respectively. Fig. 10 highlights the dynamic range of sound pressure levels inside the waveguide, represented by the distance between peaks and dips, depending on the surface impedance condition at the right end of the waveguide. With such a representation, the sound absorption performance of the termination can be visualized on the area between the maxima and minima envelopes. The smaller the area is, the more efficient the sound absorption is.

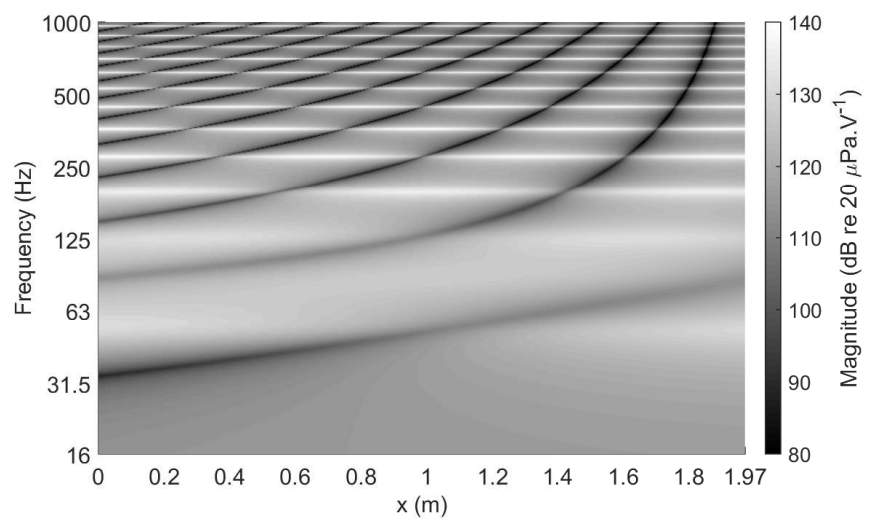

Fig. 9. Space-frequency map of the transfer function from the driving voltage of the sound source located at the left end of the waveguide to the sound pressure level. At the right end is imposed an electroacoustic absorber in open circuit (case A).

\section{ACKNOWLEDGMENT}

This research was supported by the Swiss Commission for Technology and Innovation (CTI) under grant agreement $\mathrm{n}^{\mathrm{o}} 14220.1$ PFNM-NM. The authors would like to thank P. Müllhaupt and P. Roe for their help in proofreading the paper.

\section{REFERENCES}

[1] H. Kuttruff, Room acoustics, 5th ed. Spon Press, 2009.

[2] J. Xu, J. Nannariello, and F. R. Fricke, "Optimising flat-walled multilayered anechoic linings using evolutionary algorithms," Applied Acoustics, vol. 65, no. 11, pp. 1009 - 1026, 2004.

[3] T. J. Cox and P. D'Antonio, Acoustic absorbers and diffusers: Theory, design and application, 2nd ed. Taylor and Francis, 2009.

[4] A. J. Fleming and et al., "Control of resonant acoustic sound fields by electrical shunting of a loudspeaker," IEEE Transaction on Control System Technology, vol. 15, no. 4, pp. 689-703, 2007.

[5] H. Lissek, R. Boulandet, and E. Rivet, "Optimization of electric shunt resonant circuits for electroacoustic absorbers," in Acoustics 2012, Nantes, France, April 23-27, 2012.

[6] A. J. Fleming, S. O. R. Moheimani, and S. Behrens, "Synthesis and implementation of sensor-less active shunt controllers for electromagnetically actuated systems," IEEE Transaction on Control System Technology, vol. 13, no. 2, 2005.

[7] R. Boulandet, E. Rivet, and H. Lissek, "Achieving sensorless broadband electroacoustic absorbers through electrical shunt impedance synthesis," to be published.

[8] W. M. Leach, "Loudspeaker voice-coil inductance losses: circuit models, parameter estimation, and effect on frequency response," J. Audio Eng. Soc., vol. 50, no. 6, 2002.

[9] K. Thorborg and A. D. Unruh, "Electrical equivalent circuit model for dynamic moving-coil transducers incorporating a semi-inductor," $J$. Audio Eng. Soc, vol. 56, no. 9, pp. 696-709, 2008. [Online]. Available: http://www.aes.org/e-lib/browse.cfm?elib=14633

[10] H. Lissek, R. Boulandet, and R. Fleury, "Electroacoustic absorbers: bridging the gap between shunt loudspeakers and active sound absorption," J. Acoust. Soc. Am., vol. 129, no. 5, 2011.

[11] J. Yuan, "Causal impedance matching for broadband hybrid noise absorption," The Journal of the Acoustical Society of America, vol. 113, no. 6, pp. 3226-3232, 2003.

[12] S. A. Lane and R. L. Clark, "Dissipative feedback control of a reverberant enclosure using a constant volume velocity source," ASME J. Vibr. Acous., vol. 120, 1998.

[13] Y. Cho, S. Wang, and K. Park, "Electroacoustic absorber using disturbance-observer-type velocity estimator," IEEE/ASME Transactions on Mechatronics, pp. 1-1, 2015. [Online]. Available: http://dx.doi.org/10.1109/TMECH.2015.2435020 


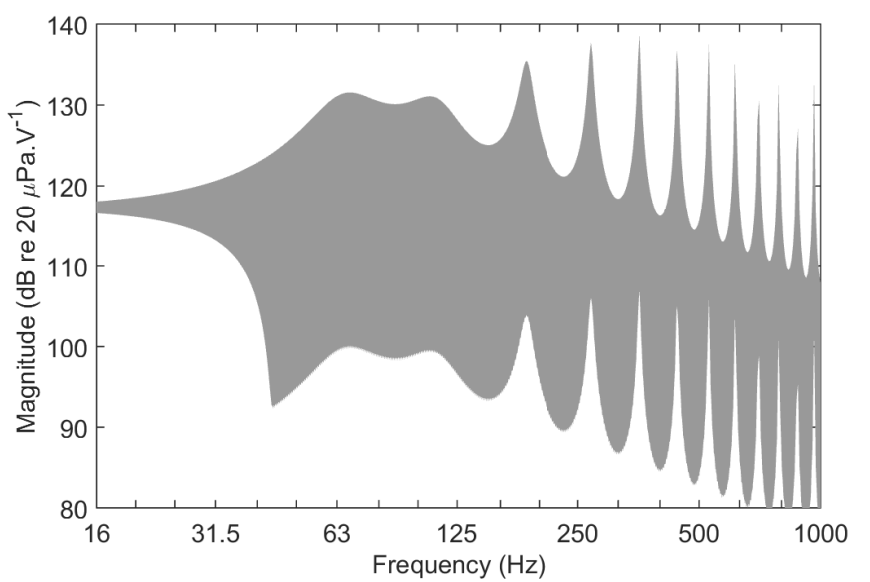

(a)

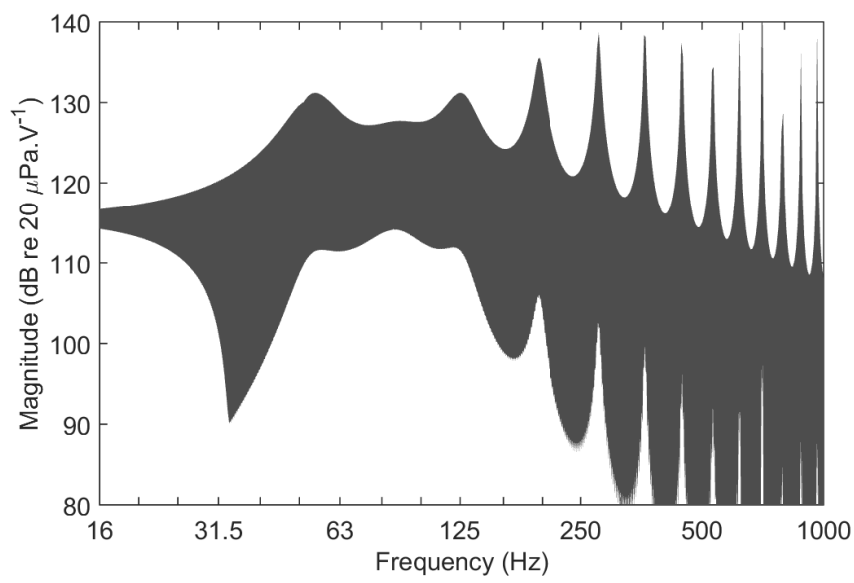

(b)

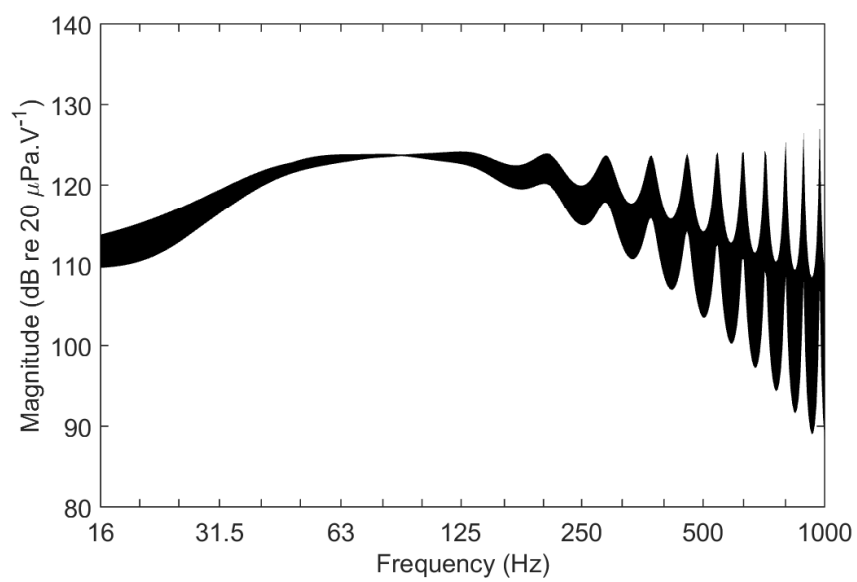

(c)

Fig. 10. Dynamic range of all the transfer functions from the driving voltage of the sound source located at the left end of the waveguide to the sound pressure level. The right end is closed by a wall (a), or by the electroacoustic absorber in cases A (b) or C (c).

[14] R. Boulandet and H. Lissek, "Toward broadband electroacoustic resonators through optimized feedback control strategies," J. Sound and Vibration, vol. 333, no. 20, pp. 4810 - 4825, 2014.

[15] V. Pagneux, N. Amir, and J. Kergomard, "A study of wave propagation in varying cross-section waveguides by modal decomposition. part i. theory and validation," The Journal of the Acoustical Society of America, vol. 100, no. 4, pp. 2034-2048, 1996.
[16] S. Karkar, E. Rivet, H. Lissek, D. Strobino, A. Pittet, V. Adam, and A. Roux, "Electroacoustic absorbers for the low-frequency modal equalization of a room: what is the optimal target impedance for maximum modal damping, depending on the total area of absorbers?" in Proceedings of Forum Acusticum 2014, Krakow, Poland, September 7-12, 2014.

[17] "Iso 10534-2-1998 : Acoustics - determination of sound absorption coefficient and impedance in impedance tubes - part 2 : Transfer-function method," ISO, Geneva, Switzerland, 1998.

[18] R. A. Pease, "A comprehensive study of the howland current pump," National Semiconductor. January, vol. 29, 2008.

[19] J. Steele and T. Green, "Tame those versatile current-source circuits," feedback, vol. 2, p. H5, 1992.

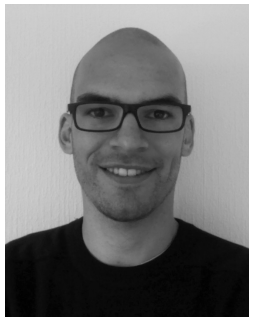

Etienne Rivet received the Dipl. Ing. degree in Electronics, Telecommunications and Computer Science at École Supérieure de Chimie Physique Électronique de Lyon, Lyon, France, in 2011. He is currently pursuing the Ph.D. degree in electrical engineering with the Ecole polytechnique fédérale de Lausanne (EPFL), Lausanne, Switzerland.

He was a research assistant in the Laboratory of Electromagnetics and Acoustics, EPFL, from 2011 to 2012, where he was responsible for several projects and supervised student projects. His current research interests include electroacoustics, low-frequency electroacoustic absorbers, room modal equalization, and sound energy harvesting.

Mr. Rivet is a member of the Swiss Acoustical Society, the French Acoustical Society, and the Audio Engineering Society.

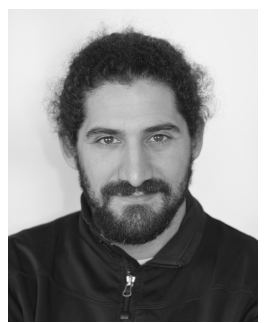

Sami Karkar received the Degree from the École Normale Supérieure de Cachan, Cachan, France, and the M.Sc. degree and the Ph.D. degree in acoustics, from Aix-Marseille Université, Marseille, France, in 2007 and 2012, respectively.

He was invloved in research on nonlinear vibrations and associated numerical methods, applied to music acoustics, with the Mechanics and Acoustics Laboratory of the Centre National de la Recherche Scientifique (CNRS), Marseille, France. In 2012, he joined the Environment and Climate Science Laboratory (LSCE) of the Comissariat à l'énergie atomique et aux énergies renouvelables (CEA), Saclay, France, where he has been invloved in entropybased climate modeling. In 2013, he joined the Signal Processing Laboratory LTS2, (EPFL), as a research assistant. He is currently a Research Associate with the Tribology and System Dynamics Lab. (LTDS) in Ecole Centrale de Lyon, Lyon, France. His current research interests include active absorption, modal control, noise control, acoustic metamaterials, and smart materials and structures involving electro-acoustic elements.

Dr. Karkar is a member of the French Acoustical Society. He passed the physics teacher credentialing exam in 2006. 


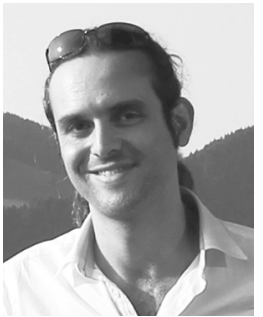

Hervé Lissek was born in Strasbourg, France, in 1974. He received the Degree in fundamental physics from Université Paris XI, Orsay, France, in 1998, and received the $\mathrm{PhD}$ degree from Université du Maine, Le Mans, France, in July 2002, with a specialization in acoustics. He is heading the Acoustic Group in the Signal Processing Laboratory (LTS2) at EPFL.

He was a Postdoctoral Researcher in the Laboratory of Electromagnetics and Acoustics at the Ecole polytechnique fédérale de Lausanne (EPFL),

Lausanne, Switzerland., from 2003 to 2005. Since 2006, he has been the Head of the Acoustic Group with EPFL, previously within the Laboratory of Electromagnetics and Acoustics (LEMA), and now in the Signal Processing Laboratory (LTS2). His current research interests include electroacoustics, active noise control, signal processing for acoustics, development of the electroacoustic absorber concept, transmission-line based acoustic metamaterials with negative/zero/positive acoustic refraction indices, and passive microphone arrays aiming at tracking mobile sound sources.

Dr. Lissek is the Vice-President of the Swiss Acoustical Society, and a member of the French Acoustical Society, the Acoustical Society of America and the Audio Engineering Society. He is also the Swiss Representative of the European Acoustics Association, the International Institute of Noise Control Engineering, the International Commission for Acoustics, and the Aeroacoustics Specialists Committee of the Council of European Aerospace Societies. 